\title{
INVERSIÓN SÍSMICA POR ALGORITMO GENÉTICO CON MIGRACIÓN DE POBLACIONES PARA ESTIMAR ANISOTROPÍA HTI
}

Juan J. Tovar ${ }^{1}$; Ovidio Almanza ${ }^{2}$; Luis Montes-Vides ${ }^{3 *}$

DOI: http://dx.doi.org/10.18273/revbol.v38n4-2016006 @) (1)

Forma de citar: Tovar, J.J., Almanza, O, y Montes-Vides, L. 2016. Inversión sísmica por algoritmo genético con migración de poblaciones para estimar anisotropía HTI. Boletín de Geología, 38(4): 107-117.

\section{RESUMEN}

Se implementó un algoritmo genético en MATLAB para realizar inversión sísmica y determinar anisotropía HTI, el cual se aplicó a un proyecto sísmico en la cuenca del Valle Medio del Magdalena en Colombia. El algoritmo que incluye el concepto de migración de poblaciones (MP), fue probado en condición de isotropía (paralelo a las fracturas) y anisotropía (perpendicular a las fracturas), mostrando mejor desempeño en la fase de prueba con datos reales. Se determinó la presencia de anisotropía débil en la zona de estudio con asociación a fracturas con acimut de $45^{\circ}$, perpendicular al régimen de esfuerzo actual en la zona, lo que explicaría la anisotropía débil relacionada con fracturas asociado a un régimen de paleo-esfuerzos.

Palabras claves: anisotropía HTI, inversión sísmica, algoritmo genético.

\section{DETERMINATION OF ANISOTROPY HTI BY SEISMIC INVERSION USING GENETIC ALGORITHM IN THE VALLE MEDIO OF MAGDALENA BASIN}

\begin{abstract}
A genetic algorithm was implemented in MATLAB to perform seismic inversion and determine HTI anisotropy, which was applied to a seismic project in the Middle Magdalena Valley basin in Colombia. The algorithm, which includes the concept of migration of populations (MP), was tested in an isotropic condition (parallel to the fractures), and in a condition of anisotropy (perpendicular to fractures), showing better performance in the testing phase with real data. The presence of weak anisotropy is determined in the study area with associated fractures oriented in azimuth of $45^{\circ}$, being perpendicular to the current stress regime. This result would explain the weak anisotropy related to fractures associated with a paleo-stress regime.
\end{abstract}

Keywords: HTI anisotropy, seismic inversion, genetic algorithm.

\footnotetext{
${ }^{1}$ Universidad Nacional de Colombia, Cra 30 № 45-03, Dpto. de Geociencias, Bogotá jjtovara@unal.edu.co

${ }^{2}$ Universidad Nacional de Colombia, Cra 30 № 45-03, Dpto. de Física, oaalmanzam@unal.edu.co

3* Autor de Correspondencia. Universidad Nacional de Colombia, Cra 30 No 45-03, Dpto. de Geociencias, Bogotá lamontesv@ unal.edu.co
} 


\section{INTRODUCCIÓN}

La anisotropía HTI (acrónimo del inglés Horizontal Transverse Isotropy) se puede asociar a la presencia de fracturas verticales en las rocas, causando una variación elíptica de la velocidad de onda $\mathrm{P}$ con el acimut (Tsvankin, 1997). En tal caso, la mayor velocidad se da en dirección a las fracturas y la menor, perpendicular a ellas. El coeficiente de reflexión al tope de medios HTI varía con el ángulo de reflexión, las velocidades de ondas $\mathrm{P}$ y S y la densidad, así como con los parámetros de anisotropía (Thomsen, 1986). Una aproximación del coeficiente de reflexión (Rüger, 1997) similar a la del caso isótropo (Fatti et al., 1994) permite la inversión de sísmica 3D y estimar la dirección de las fracturas, velocidades y parámetros de anisotropía en cada CDP.

El esquema de inversión se fundamenta en los algoritmos de optimización global, con restricciones al espacio de búsqueda según el problema a solucionar, que en este caso consisten en limitar la solución a valores con sentido desde el punto de vista geológico.
Se creó un algoritmo basado en principios evolutivos implementado en MATLAB, que permite introducir conceptos de la naturaleza y probarlos para mejorar su desempeño. Se introdujo y probó un concepto nuevo llamado migración de poblaciones (MP), demostrando un buen desempeño en sismogramas sintéticos, sísmica de campo y registros de pozo. La aplicación de estas técnicas permitió un estudio al tope de la Formación La Paz en la Cuenca del Valle Medio del Magdalena (VMM), cuyos resultados se integraron con información geológica del área.

\section{MARCO GEOLOGICO}

La zona de estudio está en el borde nororiental de la cuenca del VMM, como se muestra en la FIGURA 1A y corresponde a una cuenca intra cordillera limitada al norte por la población del Banco (Magdalena) y la falla de Bucaramanga, al sur por la falla de Cambao, al oriente por la falla La Salina y al occidente por el sistema de fallas de la cordillera Central. El procedimiento se aplicó a un área de $4 \mathrm{~km}^{2}$.
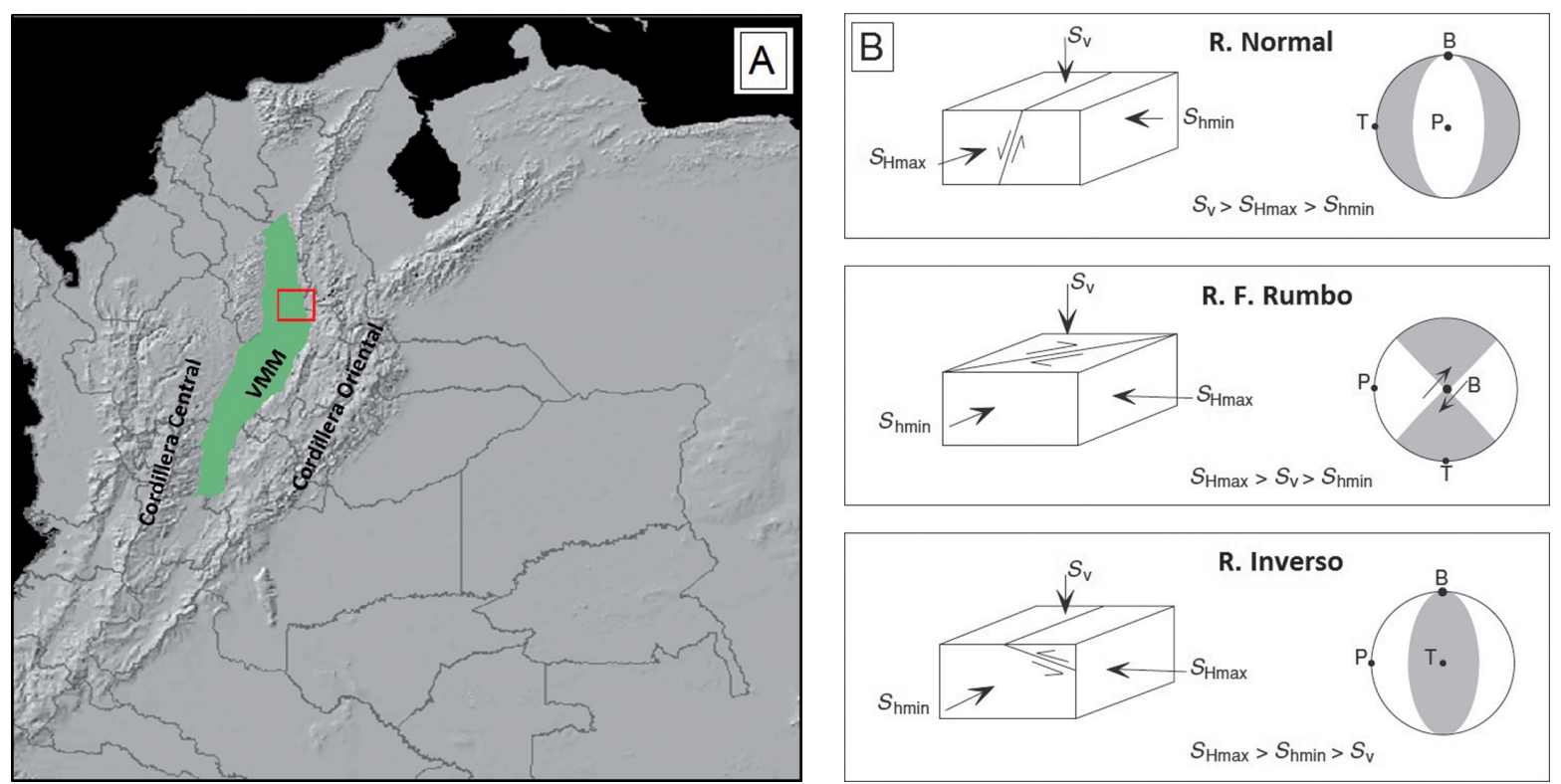

FIGURA 1. A. Contexto geográfico de la zona de estudio, la cual se encuentra delimitada por el recuadro al NE de la Cuenca del Valle Medio del Magdalena VMM entre las cordilleras Central y Oriental. B. Clasificación del régimen de falla y estado de esfuerzos asociados de acuerdo a E.M. Anderson (Zoback, 2007)

Desde el punto de vista estructural, la cuenca del VMM ha sido afectada por episodios distensivos y compresivos. La etapa distensiva comprendida entre el Triásico Tardío y el Cretácico Superior se caracteriza por la formación de un graben supra-continental, formación de fallas normales y subsidencia por causa de una tectónica de bloques (Fabre, 1983), permitiendo la acumulación de sedimentos continentales de las formaciones Bocas,
Jordán, Girón y Los Santos. En esta etapa de distensión ocurre vulcanismo, evidenciado por intercalaciones piroclásticas en la Formación Jordán. Durante el Cretácico se presenta una transgresión general que dura hasta principios del Paleógeno. La regresión está marcada por la depositación de la Formación Lisama. La fase de tectónica compresiva se inició en el Paleoceno y continúa en la actualidad (Mojica y Franco, 1990). 
Desde el Maastrinchtiano al Paleoceno se produjo el acortamiento y levantamiento de la Cordillera Central por acción de deformación compresiva (Cooper et al., 1995). El anterior evento compresivo creó una extensa cuenca de ante-país asociada a la Cordillera Central hasta el Escudo de la Guayana (Caballero et al., 2010), dentro de la cual se depositaron sedimentos de ambientes continental en el Eoceno. Desde el Eoceno hasta el Mioceno se depositan las formaciones La Paz, Esmeraldas, Mugrosa y Colorado en ambientes continentales. Durante el Mioceno medio a tardío irrumpe una fase de compresión mayor y los sedimentos hasta entonces depositados son plegados y fallados. Las paleo-fallas normales del sócalo formadas en la fase de distensión son reactivadas como fallas inversas durante el Mioceno y Plioceno sobre las cuales la cordillera Oriental pudo cabalgar hacia el occidente. Durante el Mioceno tardío a Plio-Pleistoceno se deposita la última secuencia molásica representada por los grupos Real y Mesa, así como depósitos recientes con poca deformación (Fabre, 1983).

\section{Campo de esfuerzo y relación con las fallas}

Debido a la etapa de deformación compresiva ocurrida desde Mioceno medio a tardío, la cuenca del VMM se caracteriza por un régimen de esfuerzo predominantemente compresivo. Esto hace que los sistemas de fallas asociados presenten unas direcciones características de acuerdo a la clasificación de E.M. Anderson (Zoback, 2007), donde podemos encontrar un régimen de fallas normal, donde el esfuerzo vertical tiene la mayor magnitud de los tres esfuerzos principales $(\mathrm{Sv})$; un régimen de falla de rumbo, donde el esfuerzo con mayor magnitud es el esfuerzo máximo horizontal (Shmax) seguido por el esfuerzo vertical (Sv); y un régimen de falla inverso, en el cual los esfuerzos horizontales son Shmax y Shmin (FIGURA 1B). En estudio de los esfuerzos horizontales en el noroccidente de Suramérica (Colmenares and Zoback, 2003) se definieron tres provincias de esfuerzos: provincia del Norte de los Andes (NA), provincia de los Andes Ecuatorianos (EA) y provincia de San Sebastián-El Pilar (SS-EP) como se muestra en la FIGURA 2. La cuenca del VMM se encuentra dentro de la provincia de esfuerzo del Norte de los Andes (NA), bajo esfuerzos compresivos W-E en la parte suroccidental de la provincia (cuenca AtratoSan Juan). Cortés and Angelier (2005) identificaron regímenes de esfuerzo E-W a WSW-ENE de contracción al Norte de los Andes desde el Cretácico al Paleoceno tardío, que luego cambió a NW-SE y finalmente a WNW-ESE en la fase tectónica andina. En la conjunción de placas Suramericana, Nazca y Caribe se definen dos regímenes dominantes: uno de compresión SW-NE por la interacción de placas Nazca y Caribe y micro placa de Panamá, y otro de compresión NW-SE por la interacción placa Caribe y bloque Norte de los Andes. En la región influenciada por la placa Caribe, que incluye el norte de la cordillera Oriental y el flanco oeste de la Central, el esfuerzo es WNW-ESE de tipo inverso, mientras en la unión de la cordillera Oriental con los Andes de Mérida, el esfuerzo es de compresión SW-NE. Por otro lado, en la región sur del Pacifico de Colombia - Ecuador el esfuerzo compresivo es aproximadamente E-W y se gira a WSW-ENE al norte.

Cortés et al. (2013) reportaron dirección de fracturas asociada a esfuerzos congruentes con informes técnicos del Instituto Colombiano del Petróleo (ICP) al analizar imágenes de pozo de la cuenca del VMM.

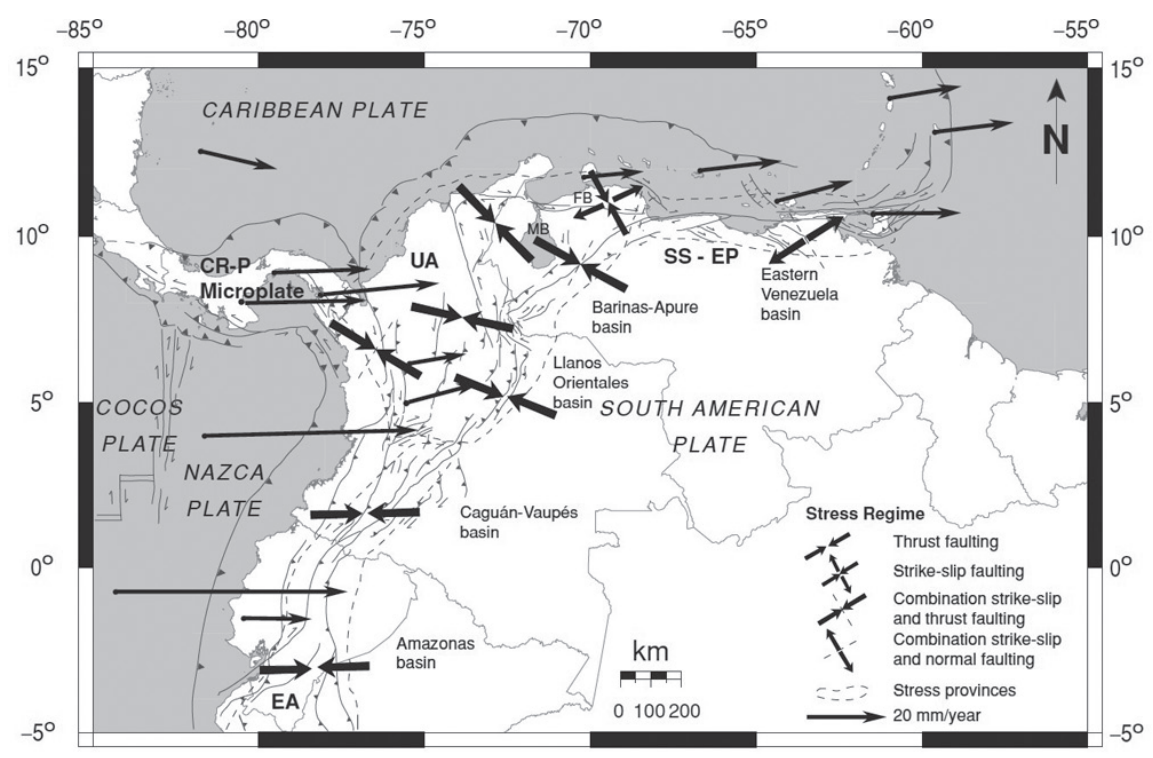

FIGURA 2. Provincias de esfuerzos en el noroccidente de Suramérica, régimen de falla y dirección de esfuerzo Shmax (Colmenares and Zoback, 2003). 


\section{ANISOTROPÍA Y ALGORITMO GENÉTICO}

En términos generales la anisotropía sísmica indica la variación direccional de las propiedades elásticas que tiene un material al paso de una onda sísmica. Convencionalmente el proceso sísmico asume un medio isótropo, sin embargo, con el advenimiento de la sísmica multicomponente, las secciones sísmicas de onda P y onda convertida CS no permitían su amarre sin considerar un modelo anisótropo. Un gran avance en la parametrización de la anisotropía fue lograda por Thomsen (1986), Alkhalifah et al. (1996), al introducir una notación sencilla e implementar un parámetro anisótropo para el procesamiento de onda P. El énfasis inicial en el desarrollo de la teoría de la anisotropía se centró en la onda $\mathrm{S}$, pero por ser la prospección con onda $\mathrm{P}$ más económica y tener un alto grado de sofisticación, muchos autores se volcaron al desarrollo en esta área. Según Crampin et al. (1985) la anisotropía sísmica se asocia a factores como: anisotropía intrínseca causada por minerales, granos o cristales alineados preferencialmente, anisotropía litológica debida a partículas de lutita o arcillas alineadas, anisotropía por capas horizontales finas de roca sedimentaria (anisotropía VTI), anisotropía por orientación preferencial de fractura o diaclasas (anisotropía HTI) y anisotropía inducida por esfuerzos.

\section{Corrección NMO en medios anisótropos}

La corrección NMO es la diferencia entre el tiempo de viaje $t_{x}$ de fuente a receptor separados una distancia $x$ (offset) y el tiempo de viaje a offset cero $t_{o}$, la cual se aplica para apilar las trazas del registro CDP (Yilmaz, 2001). En un medio isótropo $t_{x}$ y $t_{o}$ se relaciona con la velocidad de apilado $V_{N M O}$ y el offset $x$ mediante la ecuación hiperbólica:

$$
t_{x}^{2}=t_{x}+\left(x / V_{N M O}\right)^{2}
$$

En presencia de anisotropía la velocidad del medio varía con la dirección de viaje de la onda. Según Tsvankin and Thomsen (1994), en un medio anisótropo con simetría radial (VTI en FIGURA 3A) su velocidad de apilado es igual a la del medio isótropo multiplicada por un factor $\sqrt{1+2} \delta$, donde el parámetro $\delta$ puede ser negativo. En un medio con anisotropía HTI (FIGURA 3B), la velocidad $V_{N M O}$ varía con el acimut $\phi$, siendo máxima $\left(V_{\max }\right)$ en dirección de las fracturas y mínima perpendicular a ellas, y en tal caso la velocidad de apilado cambiará según:

$$
V_{N M O}=V_{\max } \sqrt{\left(1+2 \delta^{V}\right) /\left(1+2 \delta^{V} \operatorname{Sen}^{2} \phi\right)}
$$

$\delta^{V}$ es el parámetro de Thomsen para un medio VTI rotado $90^{\circ}$, y como es negativo en la mayoría de los casos, $V_{N M O}$ posee un comportamiento elíptico con $\phi$, donde el eje mayor de la elipse coincide con la dirección de las fracturas y el eje menor con el eje de simetría, lo cual permite determinar la dirección de las fracturas.
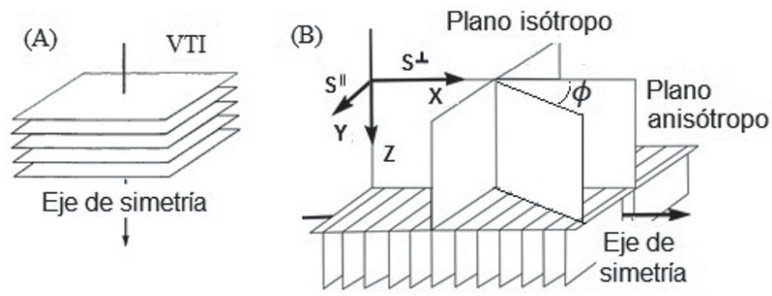

FIGURA 3. A. Modelo de medio anisótropo VTI con eje de simetría perpendicular a las capas. B. Medio HTI con el plano isótropo y el anisótropo, a un ángulo $\phi$ el plano por donde viaja la onda desde la fuente al receptor. $\mathrm{S}^{\|}$y $\mathrm{S}^{\perp}$ indican direcciones paralelas y perpendiculares a dirección de fracturas.

\section{Análisis AVO en medios isótropos y con anisotropía HTI}

En un medio isótropo, el coeficiente de reflexión $R(\theta)$ depende del ángulo de reflexión $\theta$, así como de las velocidades $V_{p}$ y $V_{s}$ y densidad $\rho$ a ambos lados del reflector, mediante relaciones analítica llamadas de Zoeppritz (1919). Una aproximación para el coeficiente de reflexión acústico muy usada es la siguiente (Fatti et al., 1994):

$$
R(\theta)=\frac{1}{2}\left(1-\operatorname{tg}^{2} \theta\right) \frac{\Delta I_{p}}{I_{p}}-\frac{4 V_{s}^{2}}{V_{p}^{2}} \operatorname{Sen} \theta \frac{\Delta I_{s}}{I_{s}}-\left[\frac{1}{2} \operatorname{tg}^{2} \theta-2 \frac{V_{s}^{2}}{V_{p}^{2}} \operatorname{Sen}^{2} \theta\right] \frac{\Delta \rho}{\rho}
$$

Donde las variables deben ser aquellas estimados en la interface: $I_{p}$ es la impedancia acústica media, $\Delta I_{p}$ es el contraste de impedancia acústica, $V_{s}$ es la velocidad media de onda $S, I_{s}$, es la impedancia de cizalla media, $\Delta I_{s}$ es el cambio de impedancia de cizalla, $\rho$ es la densidad media y $\Delta \rho$ es el contraste de densidad. La ecuación 3 se usará en el proceso de inversión sísmica para determinar $V_{p}, V_{s}$ y $\rho$ de las capas en dirección del plano de simetría. En un medio anisótropo $R(\theta)$ depende de la dirección de la reflexión, y para medios HTI, Rüger (1998) desarrolló la siguiente aproximación:

$$
\begin{aligned}
& R_{P}(\theta, \phi)=\frac{1}{2} \frac{\Delta Z}{Z}+\frac{1}{2}\left\{\frac{\Delta V_{P_{v}}}{V_{P v}}-\left(\frac{2 V_{S v}}{V_{P v}}\right)^{2} \frac{\Delta G}{G}+\left[\Delta \delta^{v}+2\left(\frac{2 V_{S v}}{V_{P v}}\right)^{2} \Delta \gamma\right] \operatorname{Cos}^{2} \phi\right\} \operatorname{Sen}^{2} \theta \\
& +\frac{1}{2}\left\{\frac{\Delta V_{P_{v}}}{V_{P_{v}}}+\Delta \varepsilon^{V} \operatorname{Cos}^{4} \phi+\Delta \delta^{V} \operatorname{Sen}^{2} \phi \operatorname{Cos}^{2} \phi\right\} \operatorname{Sen}^{2} \theta \operatorname{Tan}^{2} \theta
\end{aligned}
$$

Donde las variables se cuantifican en la interface: $\rho$ es densidad, $V_{P_{\nu}}$ es la velocidad vertical de onda $\mathrm{P}, V_{S v}$ es la velocidad vertical de onda $S, G=\rho V_{S v}{ }^{2}$ es el módulo 
de cizalla en dirección vertical, es impedancia acústica vertical, $Z=\rho V_{P_{\nu}}$ es un parámetro de separación de onda $S$ y $\varepsilon^{V}$ es un parámetro de separación de onda $\mathrm{P}$ rotado $90^{\circ}$. En la ecuación 4, el signo $\Delta$ indica cambio y Z, G, $\rho, V_{p}, V_{s}$ representan valores medios.

\section{Algoritmo genético}

Basados en la emulación del proceso natural de la evolución, cuyo principio subyacente es la supervivencia del más apto, los AG usan los operadores de Cruce y Mutación para explorar el espacio de soluciones, combinando soluciones aptas y realizando saltos evolutivos a zonas no exploradas (Mitchell, 1999). Los parámetros del modelo, en este caso velocidades de ondas P S y la densidad, son codificados en binario (cromosomas). La estructura del AG se muestra en la FIGURA 4 que explica la secuencia de pasos hasta obtener la solución. En el comienzo se genera aleatoriamente una población inicial de $\mathrm{n}$ miembros con valores de velocidades de ondas $\mathrm{P}$ y $\mathrm{S}$ y densidad limitados a intervalos previamente establecidos. Para cada individuo (solución o modelo) se generan las curvas AVO de cada reflector, usando la ecuación 3 en el plano de dirección a las fracturas y la ecuación 4 en el plano del eje de simetría (FIGURA 3B), y se comparan con los datos reales (curva AVO de cada reflector recolectado en los registros CDP). La diferencia entre la curva teórica y la real se estima como un error cuadrático medio. Esta población inicial se somete a los mecanismos de la evolución: Selección, Cruce y Mutación, produciendo modelos mejor adaptados en cada generación. Si bien estos mecanismos de la evolución operan en la dirección de mejorar las características de sus miembros, se tiene el inconveniente que si la población inicial no es muy apta transmitirá estas características a las generaciones futuras. Esto generará poblaciones homogéneas que tenderán a estabilizarse en cuanto a las características de los miembros, lo que ha ocurrido en la naturaleza en poblaciones que quedan aisladas geográficamente. Contrario a lo anterior, poblaciones que entran en contacto con poblaciones foráneas aumentan su acervo genético para futuras generaciones y con ello trae mayores posibilidades de encontrar miembros mejor adaptados. Por ende, el concepto de migración de poblaciones (MP) se ha introducido en este $\mathrm{AG}$ integrando la población foránea, que es generada aleatoriamente, como se aprecia en la FIGURA 4. El concepto de migración de poblaciones se conoce como flujo genético en los estudios de genética de poblaciones (Herrera-Paz, 2013) como un mecanismo de la naturaleza que conlleva al aumento en la diversidad del acervo genético de las poblaciones aisladas, y como tal se introdujo para modificar el comportamiento del algoritmo genético aquí implementado. No se conoce referencia que incluya está idea para algoritmos genéticos.

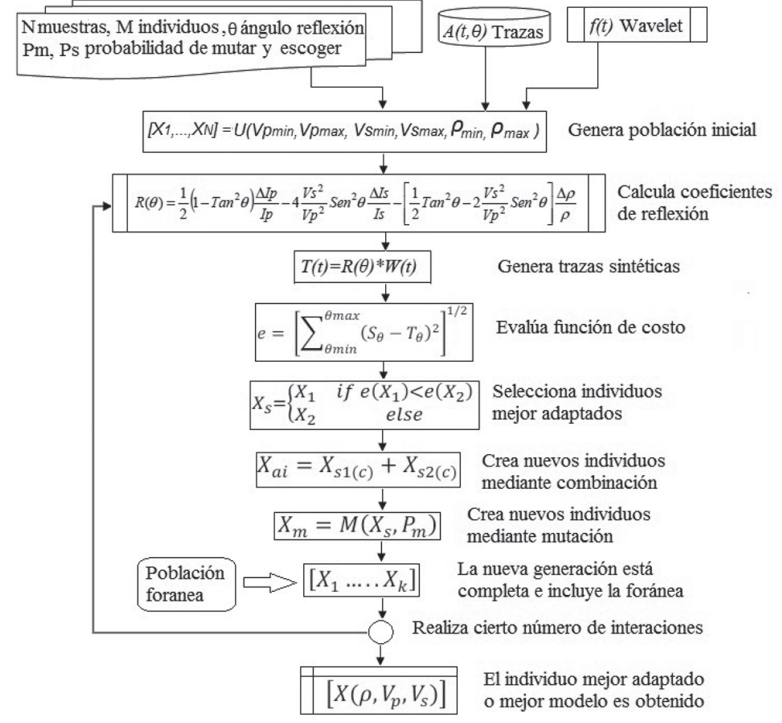

FIGURA 4. Diagrama de flujo del algoritmo genético que incluye la generación e inclusión de la población foránea en la nueva generación a fin de aumentar la diversidad genética en esta última.

\section{PRUEBAS AL ALGORITMO}

Se usó el registro gamma ray para identificar los topes de las unidades de interés e identificar en tiempo los reflectores asociados a estos topes en los registros sónico, dipolar y densidad. Para ello se amarró la imagen sísmica al pozo usando una curva tiempo-profundidad. La FIGURA 5 muestra el amarre de estos topes con los reflectores sísmicos a lo largo del pozo. Para comparar el desempeño del AG con y sin MP se hicieron pruebas con datos reales que incluían registros sísmicos y de pozo (FIGURA 6A), permitiendo identificar seis reflectores, aquí denominados R1, R2, R3, R4, R5 y R6, y sus tiempos asociados, como lo muestra la FIGURA 6B.

En un registro CDP cercano al pozo se procedió a identificar estos seis reflectores para medir las amplitudes a diferentes distancias fuente-receptor y compararlas con las amplitudes estimadas por el proceso de inversión. La FIGURA 7A muestra el comportamiento de la amplitud con la distancia fuente a recepto ("offset") medidos en el registro CDP para el reflector más somero (indicado con 'o') y las amplitudes estimadas por la inversión realizada por el $A G$ con MP (en '+') y sin MP (‘*’). Estas dos últimas poseen una forma suavizada distinta a las observadas en las amplitudes medidas, ya que aunque los registros CDP son sometidos a procesamiento permanece en ellos un error aleatorio residual. Sin embargo, se evidencia que las curvas de amplitudes estimadas por el AG siguen la tendencia de la curva de amplitudes medidas. 
$\mathrm{Al}$ comparar el ajuste entre las curvas estimadas por el AG y los datos medidos se halló que la curva MP tiene un error del 8,2 \% y un coeficiente de correlación de 7,9 mientras la sin MP un error 11,4 y un coeficiente de correlación de 6,2. En los cinco reflectores más profundos las curvas con MP tenían errores menores y mayores coeficientes de correlación comparadas con las curvas sin MP. Las FIGURAS 7B, 7C, 7D, 7E y 7F muestra las curvas de amplitudes medidas ('o') y las estimadas por el AG con MP ('+') y sin MP ('*'). Las curvas con MP y sin MP muestran tendencias diferentes sin embargo en general en la vecindad de la curva de amplitudes medidas.

De lo anterior se deduce que el AG con MP encuentra una solución con mejor ajuste con la curva de amplitud real evidenciando un mejor desempeño en la inversión.
Los resultados de la inversión por ambos algoritmos se muestran en la FIGURA 8. Al realizar una comparación de los modelos obtenidos por medio de la inversión con migración de población (azul) vs sin migración de poblaciones (roja) se aprecia que en la parte superior del registro no existe una diferencia muy notable, sin embargo al definir el paquete arcillosos del intervalo (1514-1659 pies) la inversión con migración de población lo hace mucho mejor. Adicionalmente, el contraste entre este paquete arcilloso y el intervalo arenoso inferior no es visto por la inversión sin migración de poblaciones. La densidad no es estimada de manera adecuada debido a que su efecto se manifiesta en las amplitudes a "offsets" lejanos y la información sísmica disponible solo contaba con trazas a "offsets" medianos y cercanos.

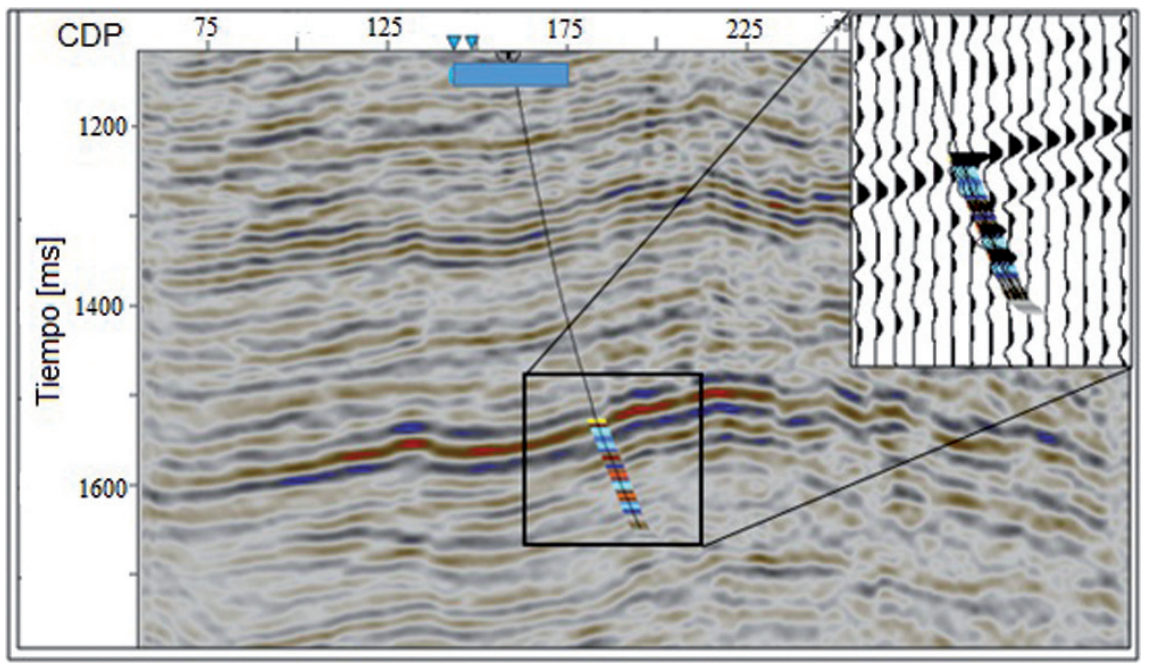

FIGURA 5. Sección PSTM con unidad geológica de interés mostrando el registro de pozo amarrado a la imagen sísmica, ampliada en el recuadro indica un buen ajuste.
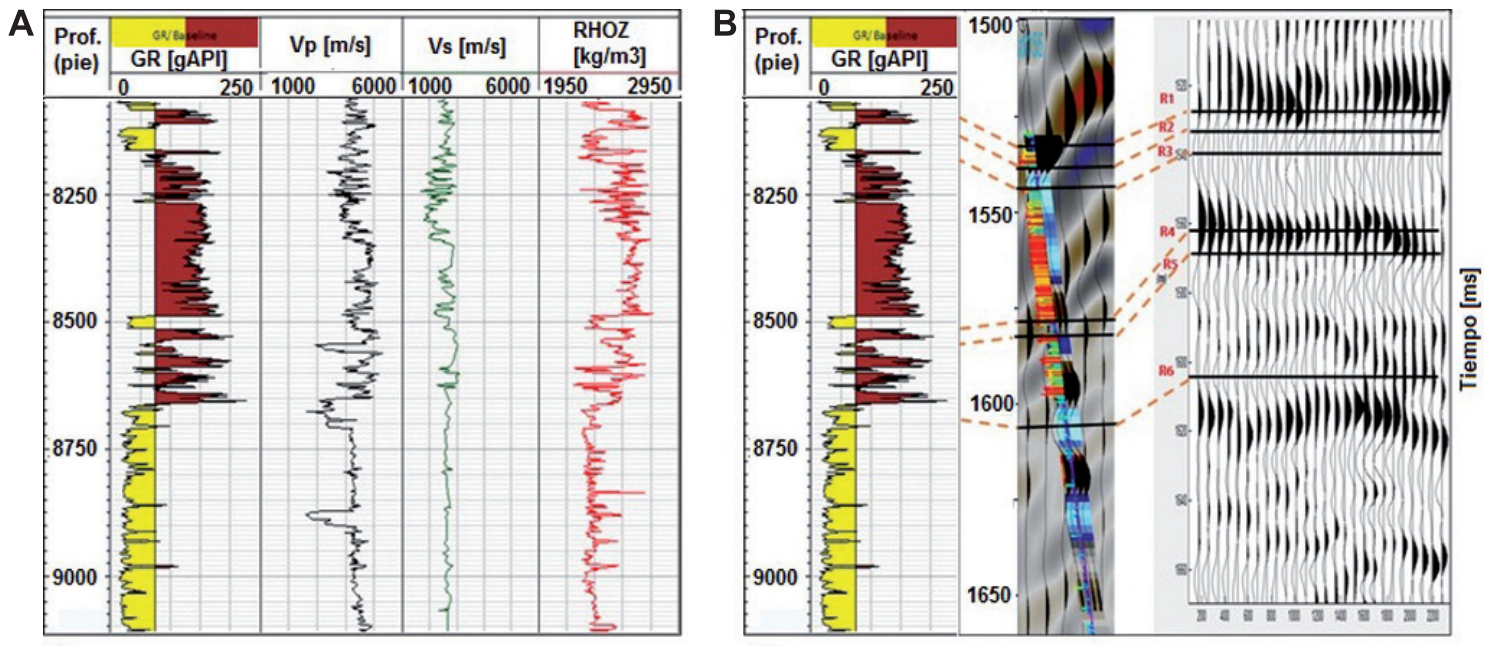

FIGURA 6. A. Sección 8210 - 9250 pies de registros de pozo gamma ray (GR), sónico (Vp), dipolar (Vs) y densidad (RHOZ) asociados a la unidad de interés. B. Registro gamma ray con topes asociados a reflectores amarrados en la sección sísmica y el intervalo 1500 y $1650 \mathrm{~ms}$ del registro CDP más cercano al pozo. 

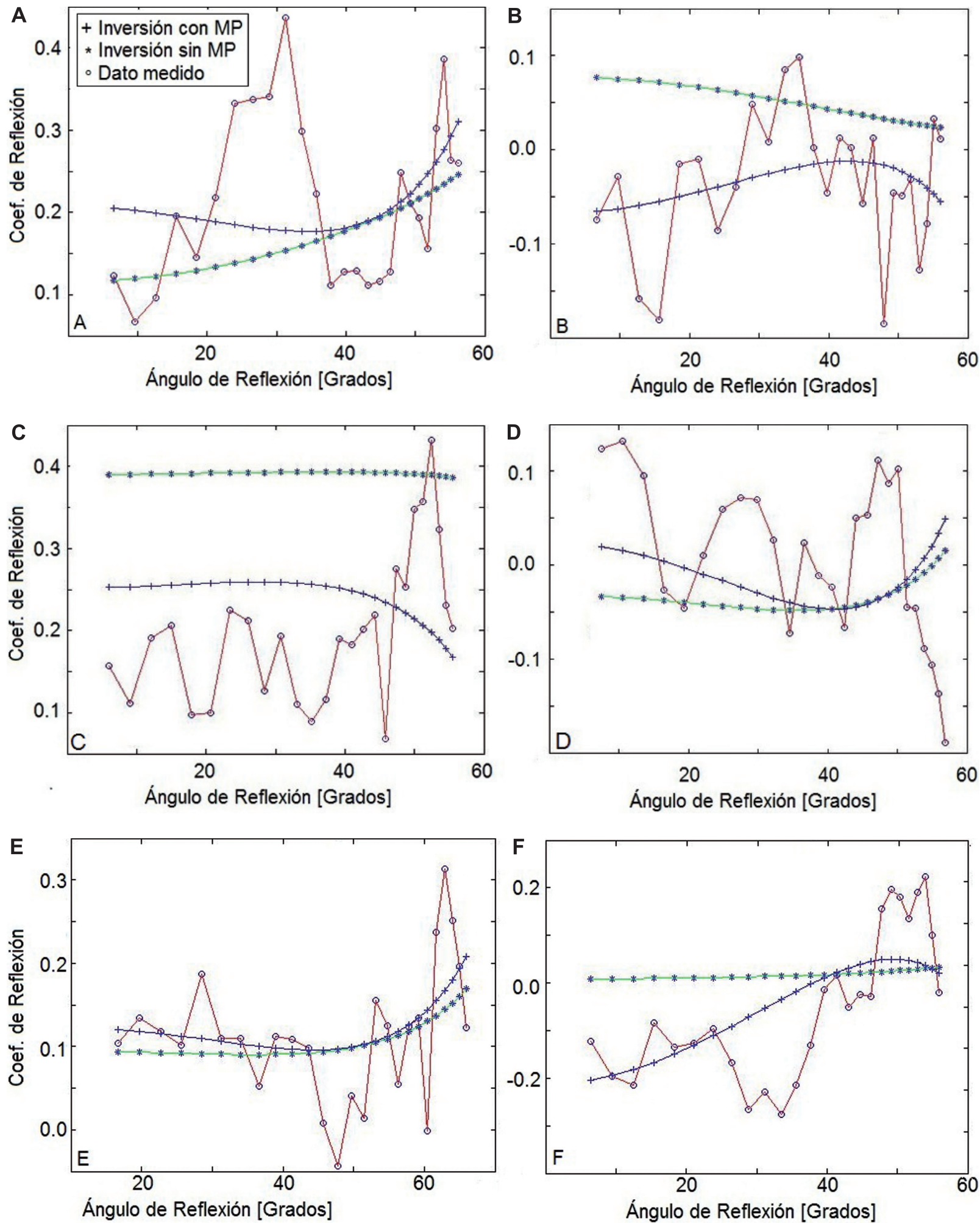

FIGURA 7. A. Curvas de amplitudes medidas en el registro CDP ('o') y las estimadas por el AG con MP ('+') y sin MP ('*') asociadas al reflector más somero R1. B. Curvas del reflector R2. C. Curvas del reflector R3. D. Curvas del reflector R4. E. Curvas del reflector R5. F. Curvas del reflector R6. 


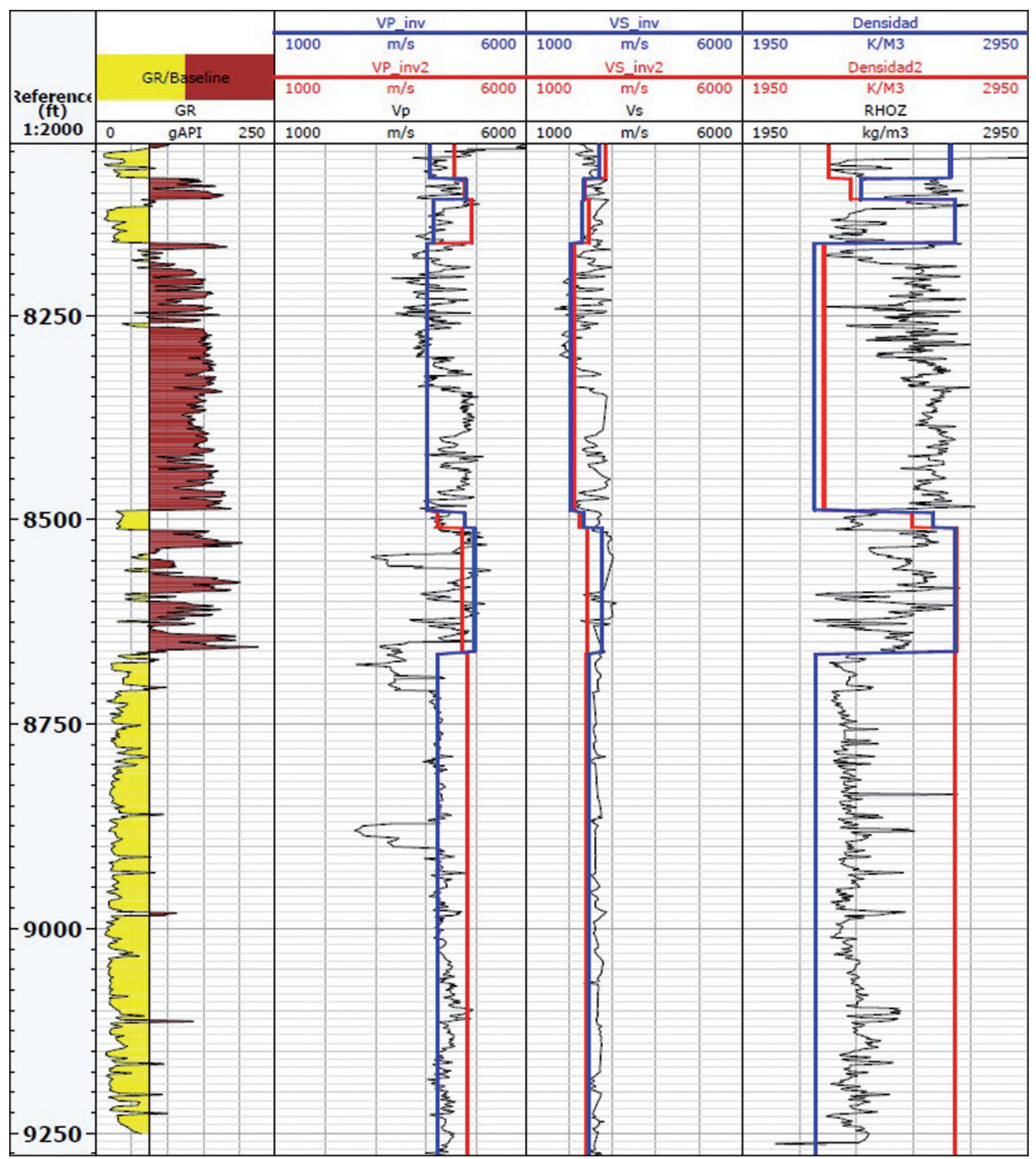

FIGURA 8. Registros sónico, dipolar y densidad, y curvas generadas (modelo de bloques) por la inversión con AG (azul) y por inversión con AG sin MP (roja).

\section{APLICACIÓN A DATOS REALES}

Se usó un volumen sísmico 3D con un cubrimiento ("fold") nominal de 60 y tamaño de cuadrícula ("bin") de $20 \mathrm{~m}$ x $60 \mathrm{~m}$, con líneas de receptores separadas 40 $\mathrm{m}$ y líneas de fuentes distanciadas $120 \mathrm{~m}$. Al volumen se le aplicó una secuencia de proceso convencional hasta la migración pre apilado en tiempo (PSTM) procurando mantener la relación de amplitudes intacta en cada reflector en los CDPs, evitando balanceo de trazas y usando filtros moderados, se hizo un análisis de velocidad para todo el volumen para obtener un cubo de velocidades inicial. Se realizó un ordenamiento de trazas por acimut ("sort") generando cuatro sub volúmenes, a saber: el primero contiene las trazas con acimut en el rango $-22,5^{\circ}$ a $22,5^{\circ}$, el segundo con trazas 
entre $22,5^{\circ}$ y $67,5^{\circ}$, el tercero con trazas entre $67,5^{\circ}$ y $112,5^{\circ}$ y el cuarto con trazas entre $112,5^{\circ}$ y $157,5^{\circ}$. Cada volumen indica una tendencia acimutal, así el primero tiene promedio $0^{\circ}$, el segundo $45^{\circ}$, el tercero $90^{\circ}$ y el cuarto $135^{\circ}$, como lo muestra la FIGURA 9E.

Para mantener el cubrimiento inicial en los nuevos cubos sísmicos se cuadruplicó el tamaño de cuadrícula generando "supergathers", garantizando que estos no superaran la zona de Fresnel. Se hizo un nuevo análisis de velocidad independiente en cada volumen generando nuevos cubos de velocidad por acimut y finalmente los volúmenes fueron migrados a PSTM.

Las FIGURAS 9A, B, C y D muestran el comportamiento de la velocidad en el reflector al tope de la unidad de interés en los volúmenes por acimut $0^{\circ}$, $45^{\circ}, 90^{\circ}$ y $135^{\circ}$ respectivamente, con la misma escala de colores para todos. Comparando la velocidad en la zona nororiental de las cuatro secciones se observa que la mayor velocidad en amarillo se encuentra en la FIGURA 9B (acimut $45^{\circ}$ ), la cual indica la tendencia direccional de las fracturas. A su vez, en la zona central predominan mayores velocidades en la FIGURA 9C (acimutal $90^{\circ}$ ), donde también se distingue un corredor que atraviesa la zona en dirección S-NW que se podría confundir con un corredor de fallas. Sin embargo, en este corredor la velocidad tiene valores cercanos para todos los acimuts indicando realmente una zona de isotropía. Es de resaltar que las zonas de interés son aquellas que cambian de velocidad (de color) con el acimut ya que así evidencian anisotropía.

Por otro lado, la zona S-E en las cuatro imágenes de la FIGURA 9 manifiesta un color fijo (cian) predominante, indicando un comportamiento isótropo. Los cubos de velocidad permiten determinar las zonas con anisotropía donde la variación porcentual de la velocidad NMO es mayor a $1,5 \%$ con el acimut y seleccionar los CDP a los cuales se les aplicará la inversión por anisotropía. En este caso, la dirección de las fracturas es al acimut $45^{\circ}$, y se procede a aplicar la inversión a la zona N-E en dos fases: primero se aplica una inversión isótropa en la dirección de las fracturas (plano isótropo) por medio de la ecuación 3 para determinar las velocidades y densidad de las dos capas (a ambos lados del reflector), y luego se aplica una inversión con anisotropía en dirección perpendicular a las fracturas (dirección de menor velocidad) por medio de la ecuación 4, para cuantificar los parámetros anisótropos $\varepsilon, \delta, \gamma$. Los resultados obtenidos se muestran en la FIGURA 10.

Los valores de anisotropía encontrados en la zona indican una anisotropía débil, la cual, de acuerdo a la FIGURA2, se debe a que la dirección de los esfuerzos tiende a cerrar las fracturas. En la FIGURA 10A predominan valores de $-0,08$ para el parámetro $\delta$, alcanzando en algunos casos a $-0,15$ (zonas moradas). Aunque la interpretación física de este parámetro no es muy intuitiva, sus valores son consistentes con los publicados por Thomsen (1986). La distribución de parámetro en la FIGURA 10B, el cual está relacionado con la velocidad de onda $\mathrm{P}$, presenta variaciones que van de pequeños valores negativos a 4\% (zonas amarillas) indicando una disminución de la velocidad de onda $\mathrm{P}$ de hasta el $4 \%$ en dirección perpendicular de las fracturas. A su vez, el parámetro indica la reducción porcentual de la velocidad de onda $\mathrm{S}$ cuando viaja perpendicularmente a las fracturas. La FIGURA 10C muestra el comportamiento del parámetro , con valores que fluctúan desde 3\% a 5\%, sin manifestar una tendencia lineal continua que infiera un corredor de fractura, por el contrario se encuentran cúmulos donde los tres parámetros poseen altos valores. Una causa probable es la presencia de enjambres de fracturas que puede ser asociado a litologías diferentes. Se observan tres tendencias en las cuales se alinean los enjambres (líneas rojas). Estas tendencias son coherentes con las direcciones de fracturas encontradas por análisis acimutal de velocidad de onda P. Si se unen estas tendencias se aprecia una semejanza con un canal.

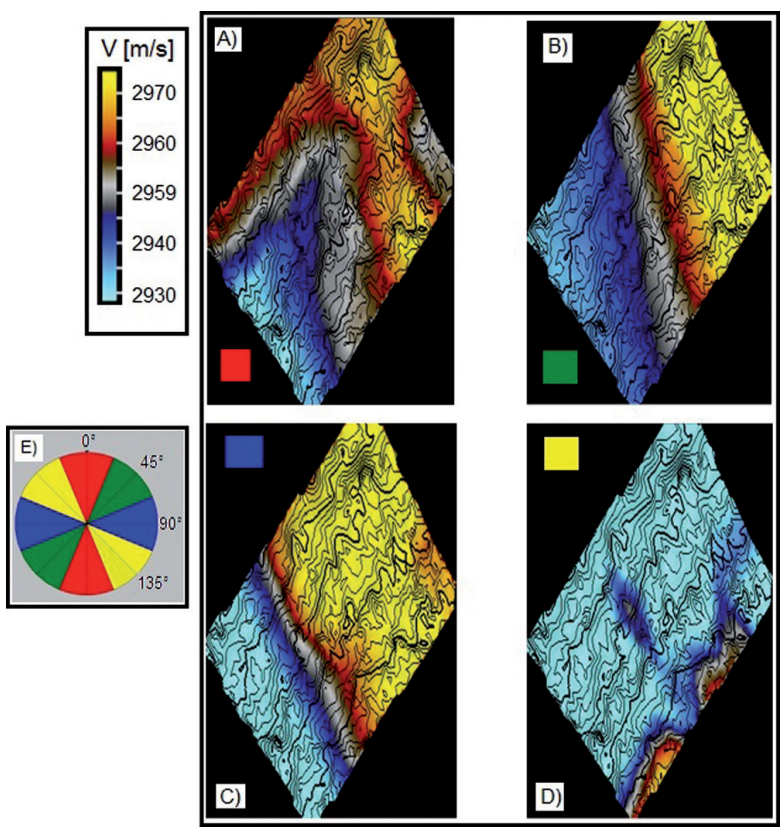

FIGURA 9. Mapa de velocidad en $\mathrm{m} / \mathrm{s}$ sobre el reflector de interés para cuatro diferentes rangos de acimut $\mathbf{A}$. acimut $0^{\circ}$, B. acimut $45^{\circ}, \mathbf{C}$. acimut $90^{\circ}$ y D. acimut $135^{\circ}$. E. Distribución de trazas por acimut: el acimut $0^{\circ}$ representa al rango de $-22,5^{\circ}$ a $22,5^{\circ}$ (rojo), el de $45^{\circ}$ representa al de $45^{\circ}$ hasta $67,5^{\circ}$ (verde), el de $90^{\circ}$ al de $67,5^{\circ}$ hasta $110,5^{\circ}$ (azul) y finalmente el de $135^{\circ}$ al de $110,5^{\circ}$ hasta $157,5^{\circ}$ (amarillo). 

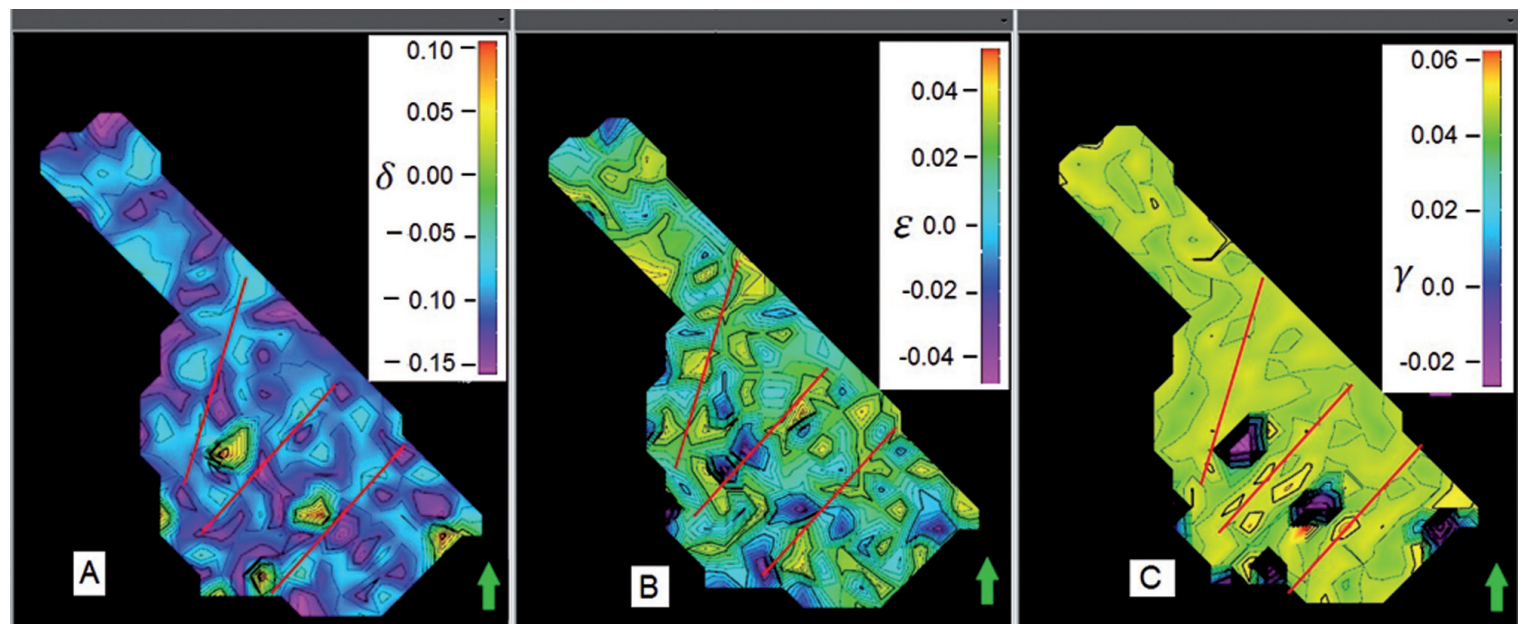

FIGURA 10. Distribución de los parámetros elásticos A. $\delta$, B. $\varepsilon$ y C. $\gamma$ suministrados por la inversión.

\section{CONCLUSIONES}

El algoritmo genético codificado en MATLAB, que incluye el concepto migración de poblaciones, mostró un mejor desempeño en la inversión sísmica al probarse con datos reales.

El procesamiento acimutal de la sísmica mostró direcciones de fracturas en acimut de $45^{\circ}$ e indicios de anisotropía débil en la zona de estudio, resultado congruente con un escenario de fracturas formadas por un régimen de paleo-esfuerzo y que están siendo cerradas por el régimen de esfuerzo actual de acuerdo a estudios regionales (Colmenares and Zoback, 2003).

La aplicación del algoritmo genético para determinar los valores de los parámetros anisótropos señala presencia de anisotropía débil y descarta presencia de corredores de fracturas, las cuales se ubican en pequeños cúmulos con conexión limitada. Sin embargo, estos cúmulos presentan alineaciones que podrían asociarse al ambiente de depositación fluvial. Esto no se pudo confirmar en este trabajo debido a la ausencia de registros de imágenes (FMI) en la zona de estudio, aunque los resultados complementarían estudios en esa dirección.

La metodología planteada en este trabajo muestra un potencial como herramienta complementaria a los análisis de ambientes de depositación, de esfuerzo y mapeo de fracturas por medio de imágenes de pozo.

\section{AGRADECIMIENTOS}

Los autores manifiestan su reconocimiento y agradecimiento a la Universidad Nacional de Colombia - sede Bogotá - y en particular al Programa de Maestría en Geofísica del Departamento de Geociencias. De manera especial damos gracias a los evaluadores del Boletín de Geología cuyas lecturas y sugerencias contribuyeron a la mayor claridad de este documento.

\section{REFERENCIAS}

Alkhalifah, T., Tsvankin, I., Larner, K., and Toldi, J. 1996. Velocity analysis and imaging in transversely isotropic media: Methodology and a case study. The Leading Edge, 15: 371-378.

Caballero, V., Parra, M., y Mora, A. 2010. Levantamiento de la cordillera Oriental de Colombia durante el Eoceno tardío - Oligoceno temprano: proveniencia sedimentaria en el Sinclinal de Nuevo Mundo, cuenca Valle Medio del Magdalena. Boletín de Geología, 32(1): 45-77.

Colmenares, L., and Zoback, M. D. 2003. Stress field and seismotectonics of northern South America. Geology, 31(8): 721-724.

Cooper, M. A., Addison, F.T., Alvarez, R., Coral, M., Graham, R.H., Hayward, A.B., Howe, S., Martínez, J., Naar, J., Peñas, R., Pulham, A. J., and Taborda, A. 1995. Basin development and tectonic history of the Llanos Basin, Eastern Cordillera, and Middle Magdalena Valley, Colombia. American Association of Petroleum Geologists, Bulletin, 79 (10): 1421-1443.

Cortés, M., and Angelier, J. 2005. Current states of stress in the northern Andes as indicated by focal mechanisms of earthquakes. Tectonophysics, 403: 29-58.

Cortés, D., Agudelo, W., and Montes, L. 2013. Estimation of density and fracture orientation in HTI 
media through azimuthal analysis of $\mathrm{P}$ wave. Ciencia, Tecnología y Futuro, 5 (3): 5-18.

Crampin, S. 1985. Evaluation of anisotropy by shearwave splitting. Geophysics, 50: 142-152.

Fabre, A. 1983. La subsidencia de la cuenca del Cocuy (Cordillera Oriental de Colombia) durante el Cretácico y el Terciario. Primera parte: Estudio cuantitativo de la subsidencia. Geología Norandina, 8: 22-27.

Fatti, J., Smith, G., Vail, P., Strauss, P., and Levitt, P. 1994. Detection of gas in sandstone reservoirs using AVO analysis: a 3D case history using Geostack technique. Geophysics, 59 (9): 1362-1376.

Herrera-Paz, F. 2013. La genética de poblaciones y el origen de la diversidad humana. Revista Médica Hondureña, 81 (1): 40-45.

Mitchell, M. 1999. An introduction to genetic algorithms. Cambridge, Mass, The MIT press, pp 6 -10.

Mojica, J., y Franco, R. 1990. Estructura y evolución tectónica del Valle Medio y Superior del Magdalena. Geología Colombiana, 17: 41-64.

Rüger, A. 1997. P-wave reflection coefficients for transversely isotropic models with vertical and horizontal axis of symmetry. Geophysics, 62 (3): 713722 .

Rüger, A. 1998. Variation of P-wave reflectivity with offset and acimuth in anisotropic media. Geophysics, 63 (3): 935-947.

Thomsen, L. 1986. Weak elastic anisotropy. Geophysics, 51 (10): 1954-1966.

Tsvankin, I. 1997. Anisotropic parameters and P-wave velocity for orthorhombic media. Geophysics, 62 (4): 1292-1309.

Tsvankin, L., and Thomsen, L. 1994. Nonhyperbolic reflection moveout in anisotropic media. Geophysics, 59 (8): 1290-1304.

Yilmaz, O. 2001. Seismic data analysis: processing, inversion and interpretation of seismic data. Second edition. Society of Exploration Geophysicists, 1000p.

Zoback, M. D. 2007. Reservoir geomechanics. Cambridge University Press, New York, pp 8-12.
Zoeppritz, K. 1919. Erdbebenwellen VII. VIIb. Über Reflexion und Durchgang seismischer Wellen durch Unstetigkeitsflächen. Nachrichten von der Königlichen Gesellschaft der Wissenschaften zu Göttingen, Mathematisch-physikalische Klasse, 66-84.

Trabajo recibido: abril 23 de 2016

Trabajo aceptado: julio 29 de 2016

Manuscrito publicado en internet: agosto 4 de 2016 